\title{
Improved Video Transmission over Mobile Ad-hoc Networks using AOMDV and MDC with Path Diversity
}

\author{
Shalini. E \\ PG Student, Department of ECE \\ Bannari Amman Institute of Technology \\ Sathyamangalam, Tamilnadu
}

\author{
T. V.P. Sundararajan \\ Professor, Department of ECE \\ Bannari Amman Institute of Technology \\ Sathyamangalam, Tamilnadu
}

\begin{abstract}
Mobile Ad-hoc Network is capable of clearing lots of challenges involved in the real-world communication. They are used in emergency response schemes, military field operation, oil drilling and mining operations. Transmitting video over MANET is an active research area due to their potential wide spread applications. In the last decade, both mobile and multimedia communications have experienced unequal rapid growth and commercial success. However, transmitting multimedia flows over wireless Ad hoc network remains an extremely challenging issue due to the limited battery lifetime of the wireless nodes. Providing good quality end-to-end video communications over mobile ad-hoc networks is more challenging due to the dynamically changing topology of the ad-hoc networks and the unreliable wireless channels. Multiple Description Coding (MDC) is an effective coding approach to enhance the error resilience of video transmission over any lossy networks. When MDC is combined with multipath transmission, MDC enables traffic dispersion and it alleviates the error propagation caused by the packet losses and hence reduces the network congestion. The error prone nature of the adhoc network always causes the frame to get corrupted. When MDC is used with such a network, it uses these corrupted frames as a reference frame and through motion compensation it compares the current frame with the reference frame and leads to error propagation throughout the network which results in video quality degradation. So, in this work, routing messages of AOMDV is used as the feedback messages after estimating the packet loss in the network and video coding is adapted accordingly with respect to the feedback messages and thus the quality of the received video is improved. AOMDV also saves the energy of the nodes by multipath routing. This work deals with comparing video quality using MDC, MDC with feedback based SMR (Split Multipath Routing) and MDC with feedback based AOMDV (Ad-hoc On demand Multipath Distance Vector routing). The experiment has been conducted using NS2 simulator along with EvalVid for evaluating the video quality. The results demonstrate that MDC with feedback based AOMDV gives good PSNR value under different packet loss rate and guarantees good video quality for large number of users in the network and at the same time the PSNR is examined by varying the number of nodes in the network resulting in decreased PSNR while trying to increase the number of nodes involved in the network.
\end{abstract}

\section{Keywords}

Ad-hoc Network (MANET), MDC (Multiple Description Coding), AOMDV (Adhoc On demand Multipath Distance Vector routing), packet loss estimation, frame corruption probability determination, Reference Picture Selection (RPS), video communication, PSNR (Peak Signal to Noise Ratio)

\section{INTRODUCTION}

Mobile Ad hoc Network (MANET) is one of the interesting and energetic area of study for the past few decades. The main reason and force for all this research work is to offer the customers with the network support at any time and also at any place. Mobile adhoc network are said to be selforganizing and self-configuring networks. No infra-structure is required to construct and manage MANETs. It works in a multi-hop style. A mobile node in MANET environment not only transmits the packets assigned to them, but also performs packet forwarding to other mobile nodes in the network. A mobile ad hoc network (MANET) consists of wireless mobile nodes dynamically forming a network topology without using any centralized administration for the purpose of communication. In recent years, there has been a growing interest in video communications over mobile wireless networks due to its plenty applications in the areas of military and other disaster relief applications with the advances of wireless networking and video coding technologies. So, providing reliable video communications over wireless ad-hoc networks faces significant challenges. The challenges arise from the dynamic changing topology of the network and the vulnerability of compressed video to packet losses. Since the transmission range of the mobile node is limited, multiple hops are required for a node to transmit its information to other node involved in the network for the purpose of energy saving. So, in MANET, routing protocols are needed to set up communication paths between nodes, without causing extreme control traffic overhead or computational load on the power constrained devices. Due to the mobility, the path between source and destination gets break down at any time and thus communication becomes a tedious problem. At the same time, since the connection is dynamic for all nodes in MANET, it is possible to set up more than one path between source and destination. So, as this property of adhoc networks is used in the routing procedure, it is termed as Multipath Routing.

Since multiple paths transmission can distribute traffic between a set of disassociate paths, it offers load equilibrising and route failure defense. So, among various error resilient techniques, Multiple Description Coding (MDC) with path diversity is found to be a hopeful method for transmitting video over lossy networks [1-2]. MDC generates multiple descriptions with equal importance while each description can reconstruct the source with acceptable quality. In wireless networks, packets suffer from bursty losses due to node's 
Video Input

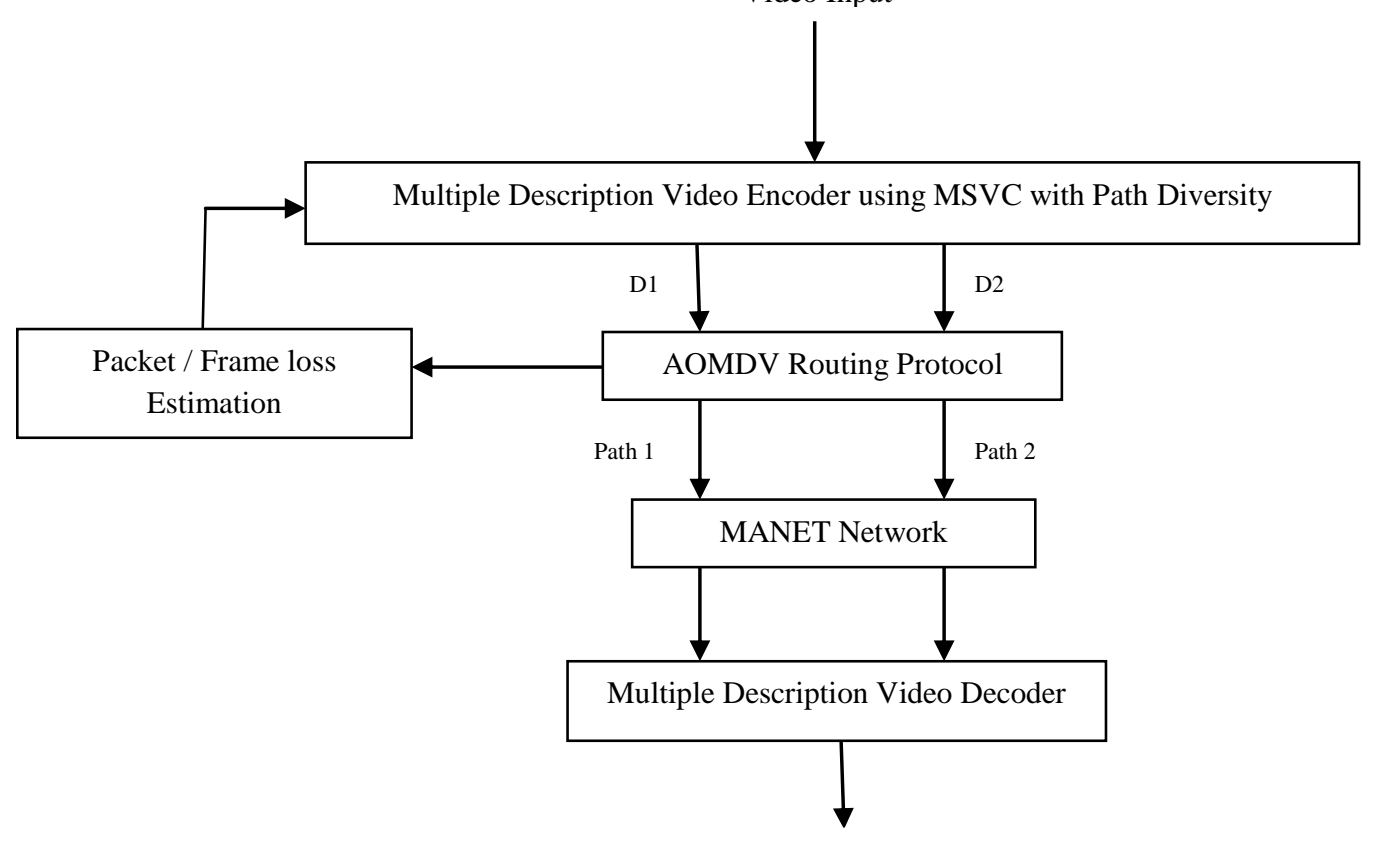

Video Output

Fig 1: Architecture of the Proposed System using AOMDV as a routing protocol and Multiple Description Coding with Path Diversity

mobility and topology changes. MDC with multiple paths is a robust technique to losses since all the descriptions are not lost simultaneously. Thus the satisfactory quality of video is maintained [3]. Also, MDC with path diversity avoids congestion in network by sharing and equilibrising the load in the network. Unlike Routing aware MDC with MPT (Multiple Path Transmission) using Split Multipath Routing (SMR) as a routing protocol [4], this proposed work uses the routing messages of Ad-hoc On demand Multipath Distance Vector (AOMDV) routing protocol in order to estimate the loss of packet and then selecting the reference frames consequently since the end to end delay is fewer and Packet Delivery Ratio (PDR) is higher in AOMDV when compare to SMR.

In this work, a model is constructed for estimating loss probability of every packet by using the routing messages of AOMDV routing protocol received by the sender, and transmission delay is found by the MAC layer access mechanisms and the parameters of the network. And finally, frame loss probability is estimated, and the reference frames are selected based on the formula (1) in order to avoid the error propagation in the subsequent frames. Unlike common Reference Picture Selection (RPS) work [5], this work not involves any additional channel for feedback about the status of the network, but it recovers information from routing messages.

The work is implemented using NS2 and EvalVid for simulation and the proposed method of video transmission over adhoc network using AOMDV achieves higher PSNR value when compare to the two methods which uses only MDC for video transmission and the other method uses SMR as a routing aware for video transmission over adhoc networks.

The paper is structured as follows. Section II describes the related work. Section III describes the architecture of the proposed work and also describes how the packet loss estimation is made based on routing message of AOMDV.
Results are described in Section IV and Section V concludes the paper.

\section{RELATED WORK}

In [6], Layer coding concept is used for video transmission, which encodes the video into two layers namely, base layer and an enhancement layer. The base layer (BL), which includes the crucial part of the video frames, guarantees a basic display quality. Each enhancement layer (EL) correctly received improves the video quality. But without the BL, video frames cannot be reconstructed sufficiently. Usually, EL packets may be dropped at a congested node to protect $\mathrm{BL}$ packets, and BL packets are better protected with FEC or ARQ [7]. These two layers are not highly correlated.

Due to this property, it is not possible to partially recover lost information of one substream, using information carried in other correctly received substreams. But MDC method overcomes this problem by generating multiple equally important streams, each giving a low but acceptable quality. A good-quality reconstruction is decodable from all bit streams received together, while a worse, but still tolerable quality reconstruction is possible only if one stream is received. The correlation among the substreams introduced at the encoder makes it possible to partially recover lost information of one substream, using information carried in other correctly received substreams. However, such a correlation limits the achievable coding efficiency, as compared with a conventional coder designed to maximize it. A key challenge with this codec is how to control the mismatch between the reference frames used in the encoder and those used in the decoder caused by transmission errors. Many approaches has been proposed in order to eliminate mismatch, but it comes at the cost of redundancy. Feedback-based video coding techniques includes tracking of error, error confinement and Reference Picture Selection (RPS). The RPS technique encodes the present video frame among reference to a earlier frame selected based on the feedback information instead of constantly using the final transmitted frame [8]. Thus, it can 
halt error propagation without causing excessive loss of coding efficiency. One of the major shortcomings of this technique is that they could be used only

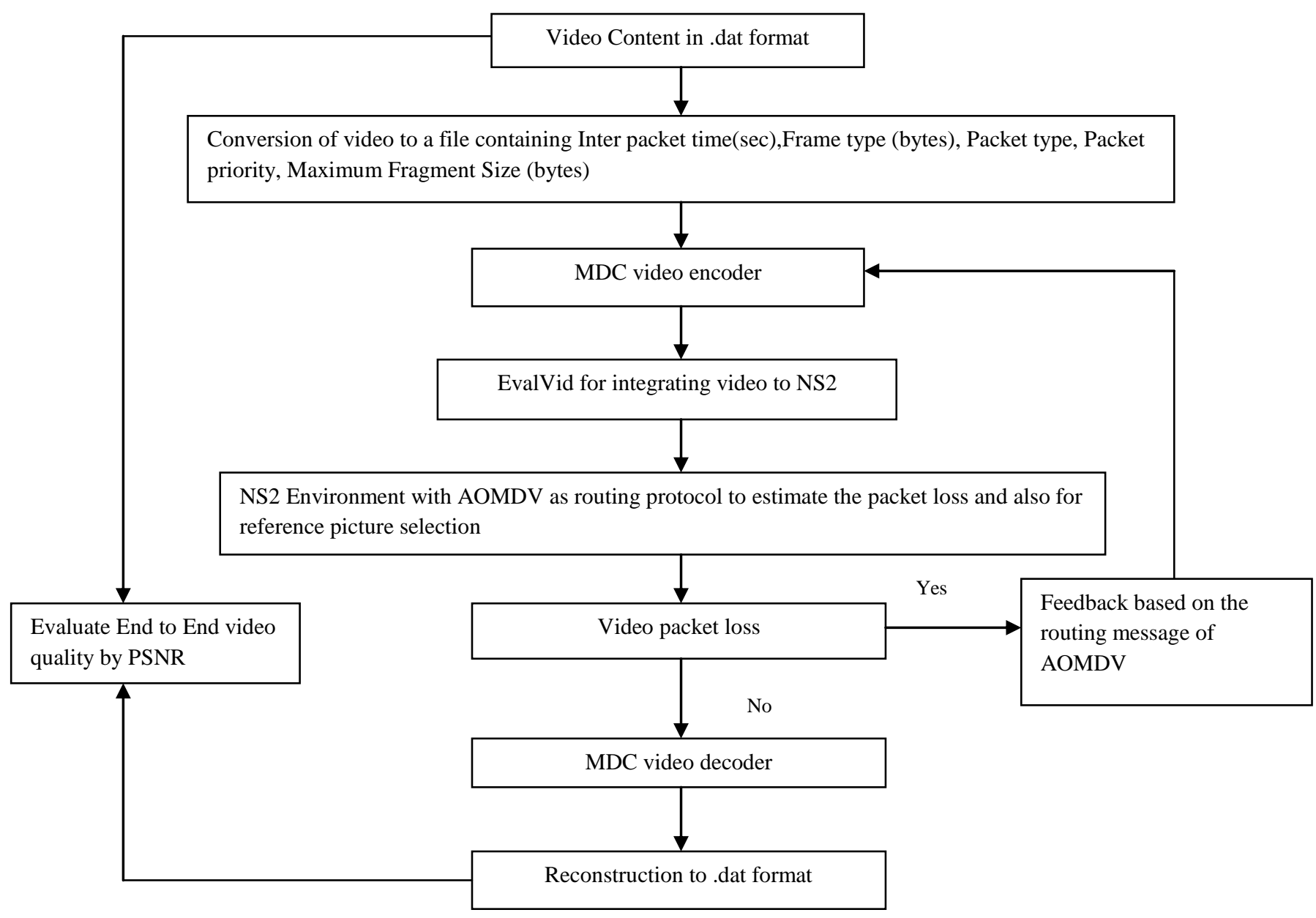

Fig 2: Layout of the proposed method

when feedback channel is present. Based on the delay associated in network for receiving the feedback information, the efficiency of the feedback based schemes are calculated. If the delay is small, the encoder can impede the error propagation, and the closer frames are utilized as reference frame. The delay in receiving the feedback information does not cause extra encoding delay or the overall end-to-end delay. Therefore, feedback-based approaches are feasible options even for interactive applications. The feedback delay seriously affects the encoding efficiency. Unlike common reference picture selection (RPS) work, the proposed work does not require any extra channel feedback but retrieves information from normal routing messages. From [9], it is observed that SMR produces more control overhead than the AOMDV routing protocols. This is caused by the fact that SMR rebroadcasts the same RREQ packets it receives from multiple neighbors. End-to-end delay for the SMR protocol is higher than AOMDV [10].

In low mobility and low node density scenarios end-to-end delay is approximately $650 \mathrm{~ms}$, while in higher mobility and node density the end-to-end delay augments to $1200 \mathrm{~ms}$. The high routing overhead of SMR penalizes data packets, therefore high buffering delays contribute to high end-to-end delay. SMR and AOMDV achieve a good dispersion of traffic between the different nodes. SMR and AOMDV have lower standard deviation than AODV multipath independent of node mobility. So, in the proposed work, AOMDV is chosen as the Routing protocol.

\section{SYSTEM ARCHITECTURE}

In the proposed method, the MDC generates two descriptions based on the Multiple State Video Coding (MSVC) [11] method since MSVC is easy to implement and it supports a variety of video standards and the Reference Frame selection is done by using the routing messages of the AOMDV routing protocol. As shown in figure 1, the MDC uses MSVC and down samples the video sequence into two sub-sequences consisting of even and odd frames and those frames are encoded into two descriptors using H.264 encoder. After encoding process, two descriptors are transmitted through two different paths by making MDC supporting MPT (Multiple Path Transmission). MDC with MPT helps in reducing the continuous loss of descriptions and balances the load in the network [12]. Thus increasing the gain of video transmission over MANET. The routing in adhoc networks is done by using AOMDV protocol. By the MAC layer mechanism, loss probability of packets is estimated and the AOMDV routing message is feedback to the MDC encoder based on this estimation, and thus the error propagation is totally eradicated in the subsequent descriptors of the collapsed route. The 
layout of the proposed work is shown in figure 2. This represents the entire flow diagram of the proposed work.

Initially, video is obtained in dat format. The multiple description (MD) video encoder which uses SMR as a routing protocol for aware routing generates two video descriptions based on the multiple state video coding (MSVC) method. The two different descriptions are transmitted through two different paths established by the multipath routing protocol. The encoded video is given as input to the NS2 using EvalVid (A Video Quality Evaluation Tool set). Ad-hoc network is constructed in the NS2 environment, which uses AOMDV as a routing protocol. If packet loss is estimated using the RERR message of the AOMDV, reference picture selection is made based on the estimated frame loss probability to retransmit the lost frames and then decoding is done by the receiver. If there is no packet loss, decoding is performed directly at the receiver by the MD decoder. Finally, PSNR value is calculated between the original video which is given as input and the reconstructed video obtained at the output.

\subsection{Multiple Description Video Encoder and Decoder}

Multiple Description Coding (MDC) is a most effectual to fight against loss of packets in internet and wireless communication networks [13]. At the same time, it has good potential for real time applications of video in which retransmission is intolerable and also not feasible. The main objective of MDC is to generate numerous descriptions that are independent which has one or more features of video such as frequency content, spatial or temporal resolution and signal-to-noise ratio.

Descriptions may have the same importance or they may have different importance. When more descriptions are received, higher the quality of decoded video. MDC encoder generates two descriptions which are sent separately across two different channels [14]. Both the descriptions are received by the MD decoder or either one of the two descriptions are received. So the decoder may be in one of the three states. The central decoder receives both the descriptions and produces good quality reconstruction. While the two side decoders can receive one among the two descriptions and produces only the lower but acceptable video quality. MSVC [15] is applied to MD video encoder. In the encoder part, by using H.264 encoder, video is encoded. Before encoding, video is temporally decimated into two sub-sequences comprising of even and odd frames respectively. At the time of encoding, routing messages from the multipath routing protocol is used to assist the encoder to choose the reference frames. At the decoder, MSVC decoder is utilized with the refined error concealment method.

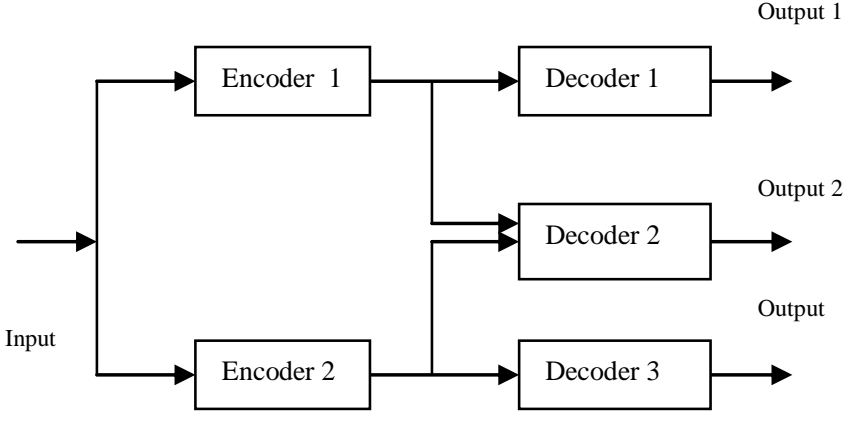

Fig 3: Multiple Description Coder

\subsection{Ad-hoc Ondemand Multipath Distance Vector Routing}

AOMDV was designed mainly for highly dynamic ad-hoc networks where link failures and route breaks occurs often. It maintains routes for destinations and uses sequence numbers to prevent routing loops. It is a timer based routing protocol and it assists mobile nodes to react to the path breaks and changes in topology. It uses three types of control messages namely route request (RREQ), route reply (RREP) and route error (RERR). A node broadcasts RREQ message throughout the network when it requires a path to send the data packets. When a node receives a RREQ packet, it checks the destination address field of RREQ. If a node has any information regarding the destination or if it is an actual destination, RREP packet is transmitted in turn to the concerned source node. If an intermediate node does not have any route to destination and optional paths are not available, it sends RERR message reverse to the upstream nodes. AOMDV routing protocol employs advertised hop count field in its routing table for several route entries. To identify multiple next hops with respect to the relevant hop counts, it uses next hop lists available in the routing table. A node updates its advertised hop count for a destination whenever it sends a route advertisement for the destination.

\subsubsection{Route Discovery Procedure}

Initially, in a route discovery procedure, a source node A primarily checks out its routing table when it has data packets for a destination in order to find out whether it previously has a route to the destination node $B$. If a route is available, it sends the data packets by utilizing its existing route. Else, it broadcast RREQ message into the network for finding out the route to the proposed destination. AOMDV provides multiple paths and observes each route advertisement to define an alternate path to the source or the destination during a route discovery procedure. RREQ packets arriving at the nodes are copied and sent back to the source nodes. This approach may lead to the formation of loops due to accepting all copied routes. In order to avoid the possibility of loops, it uses advertised hop count field in the routing tables. The advertised hop count of a node $\mathrm{S}$ for a destination $\mathrm{D}$ is set the maximum hop count of the multiple paths for $\mathrm{D}$ at $\mathrm{S}$. The advertised hop count is initialized each time the sequence number is updated. By doing so, AOMDV only accepts alternative routes with lower hop counts. Each RREQ conveys an additional first hop field to indicate the first neighbor of the source node. The intermediate nodes do not cast out duplicate copies of RREQ instantly until each RREQ render a new disjoint path to the source. If an intermediate node offers a new path, a reverse path is set up. It sends back a RREP to the source. At the destination, reverse routes are established like in the same situation of intermediate nodes.

\subsubsection{Sequence Number Procedure}

To prevent the formation of routing loops and to select the most recent routing path to the destination, a sequence numbering technique is exploited. AOMDV sets its destination Sequence Number as follows.

if

(sequence num $_{i}^{d}<$

sequence num $\left._{j}^{d}\right)$ or $\left(\left(\left(\right.\right.\right.$ sequence num ${ }_{i}^{d}=$ sequence num $\left._{j}^{d}\right)$ and (hopcount $t_{i}^{d}>$ hopcount $\left._{j}^{d}\right)$ )

then 
sequence num $_{i}^{d}=$ sequence num $_{j}^{d}$;

hopcount $_{i}^{d}=$ hopcount $_{j}^{d}+1$

nexthop $=j$;

end if

Each RREQ conveys an additional first hop field to indicate the first neighbor of the source node. The intermediate nodes do not discard duplicate copies of RREQ immediately as long as each RREQ provides a new node-disjoint path to the source. If an intermediate offers a new path, a reverse path is set up. It sends back a Route REPLY (RREP) to the source. At the destination, reverse routes are established like in the situation of intermediate nodes. If a link break occurs between a source and destination, a node that knows this link break needs to send Route Error (RERR) message back to the source node. For periodic route updates, HELLO messages are broadcast in a timely manner.

\subsubsection{Route Maintenance Procedure}

Route maintenance in AOMDV is a similar to AODV route maintenance with little extension. Like AODV, AOMDV also uses RERR packets. A node forwards a RERR control packet to a destination when the path to the destination breaks. AOMDV includes optimization to recover packets forwarded over failed links by forwarding them again over an alternate path. This is similar to the packet salvaging mechanism in DSR. The timeout mechanism similarly extends from a single path, but even though, setting proper timeout values is more difficult for AOMDV compared to AODV. With multiple paths, the paths become stale more likely. But using little timeout values to avoid stale paths can bound the benefit of using multiple paths. From [16], it is observed that AOMDV outperforms SMR in many aspects like less control overhead, less end to end delay and good packet delivery ratio. So AOMDV is chosen in this work for routing aware purpose.

\subsection{Packet Loss Estimation}

AOMDV routing mechanism states that, when the retransmission fails while transmitting a packet to the following hop by the MAC layer, then automatically the initiation of RERR message commences. This RERR message shows that a link turns unreliable and the packets transmitted through this link experience heavy rate of packet loss. When the video packets are transmitted from the source node before the reception of RERR message by the source node, the video packets are vulnerable to heavy packet losses since they are still transmitted only through the unreliable link. As the source receives the RERR, it either starts the route recovery process to find a new route or rebuilds the route from the route cache. Packets that are about to be transmitted in the cracked route during the route recovery process are thrown away and marked as missed.

The retransmission delay of the video packet to the next hop is defined as $T_{\text {retrans }}$. After time $T_{R E R R}$, source node receives the RERR message and halts the video packet transmission through the unreliable link. The other packets which are sent during this time period are however transmitted only through this unreliable link and are vulnerable to heavy packet losses.
So in [17], they presume that anytime the source receives an RERR, the preceding video packets sent from the source track the same packet loss distribution under the same network conditions. Before receiving an RERR message by the source node, the loss probability of the leading packets from the source node is denoted as $P_{r}(n) . P_{r}(n)$ is utilized to decide the possible corrupted frames. Because of the stochastic delay existing between link failure and RERR message reception at the source, the $n$-th preceding packet before RERR can be sent at a time earlier, right at, or later than the link failure occurs. These three cases are represented by the three states namely GOOD, FAIL and BAD. Packets that are sent earlier to the link breakage enters GOOD state, and those packets that fails to send and activates RERR enters FAIL state, and finally, those packets that are transmitted after the link breakdown enters the BAD state. The frame's corruption probability can be determined by estimating the loss probabilities of packets and it is given below as,

$p\left(f_{k}\right)=1-\prod_{v_{i} \in f_{k}}\left(1-p\left(v_{i}\right)\right)$

where $p\left(v_{i}\right)$ is the packet loss probability for packet $v_{i}$ in frame $f_{k}$, and the frame corruption probability of $f_{k}$ is defined by the probability that any packet in frame $f_{k}$ is lost. This frame error probability is employed for the purpose of Reference Frame Selection

\section{SIMULATION RESULTS AND DISCUSSION}

\subsection{Implementation and simulation set up of the proposed work}

The work is simulated by using both the NS2 and EvalVid and the comparison of proposed work is done with simple MDC and MDC with SMR with various network conditions. Initially the Video is obtained in .dat format. In this work, Video64.dat is chosen as a Video file. This video file is converted to a file named as video_input consisting of Inter packet time (sec), Frame size (bytes), Packet type, Packet priority, Maximum Fragment Size (bytes) by using the mpeg trace converter since NS2 does not support real time video. Video_input file is further encoded by MDC coding and is given as input to the EvalVid. For integrating EvalVid in NS2, the following changes have to be made in NS2 Make file.

1. Add myevalvid/myudp.o, myevalvid/myevalvid_sink.o and myevalvid/myevalvid.o in the OBJ_CC list in the Makefile of NS2

2. Add extra coding lines in packet.h, agent.h, agent.cc of the Makefile in order to support Inter packet time (sec), Frame size (bytes), Packet type, Packet priority, Maximum Fragment Size (bytes) in NS2

3. Recompile the Makefile

Video_input file is attached to the source node by using the agent and the video is transmitted over the Ad-hoc network. The Simulation environment is shown below in the following table. 
Table 1: Simulation Parameters

\begin{tabular}{|c|c|}
\hline Number of nodes & $50,60,70,80$ \\
\hline Video file & Video64.dat \\
\hline Region & $500 \mathrm{~m} * 500 \mathrm{~m}$ \\
\hline Routing protocol & AOMDV \\
\hline MAC Layer protocol & $15 \mathrm{~dB}$ \\
\hline Transmission Power & \begin{tabular}{c} 
(1 Kb/s) \\
\hline Traffic type
\end{tabular} \\
\hline Mobility Model & $\begin{array}{c}\text { Random Waypoint } \\
250 \mathrm{~m}\end{array}$ \\
\hline Node transmission range & $\begin{array}{c}352 \text { X } 288 \text { (CIF) } \\
\text { Video Resolution }\end{array}$ \\
\hline Radio Model & $\begin{array}{c}\text { Two ray ground propagation } \\
\text { model }\end{array}$ \\
\hline
\end{tabular}

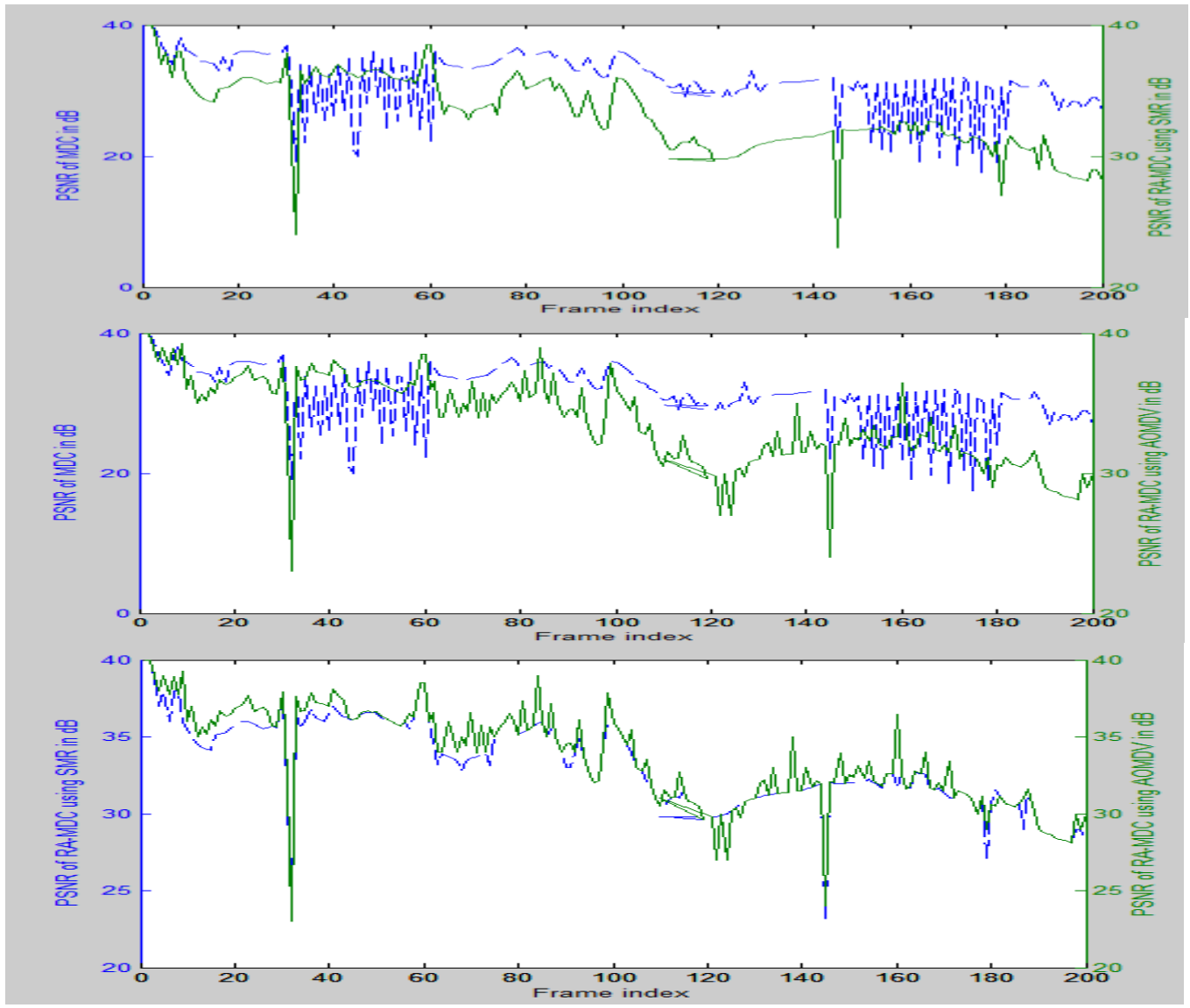

Fig 4: Comparing MDC, RA-MDC using SMR and RA-MDC using AOMDV Based on PSNR

The frames that are divided as even and odd frames are encoded using the H.264 encoder in MDC coding. The output of MDC coders are termed as descriptors. In this work, two descriptors are generated and transmitted over multiple paths in the Ad-hoc network which uses Multipath routing protocol (AOMDV). The mobile adhoc network with two path transmission is simulated using NS2. The Play out deadline indicates that the packets are dropped if it does not reach the destination with $350 \mathrm{~ms}$. The packet loss probability in a network is estimated using the MAC layer parameters, routing information, and the packet loss estimation model and this information is given to the encoder for reference frame selection using the Routing Message of AOMDV routing protocol. The selected reference frame is now compared with the current frame for motion compensation. IEEE 802.11b which employs CSMA/CA as the MAC layer protocol chosen. 
PSNR is one of the most common subjective parameter used to evaluate video quality. The following equation shows the definition of PSNR.

$$
P S N R=10 \log _{10}\left[\frac{255^{2}}{M S E}\right]
$$

The frame corruption probability is estimated using the formula (1). This frame corruption probability is utilized for the purpose of Reference Frame Selection to improve the video quality. The destination node is attached by the null agent with that of the myevalvid_sink from which output is obtained and stored in video_output file. The output file is again decoded using MDC decoder. PSNR is calculated between input video and received video in order to evaluate end to end video quality. MOS (Mean Opinion score) is a subjective metric to measure digital video quality at the application level. This quality metric is generally given on a scale ranging from 1 (worst) to 5 (best). In this work, PSNR of every frame can be approximated to the MOS scale as shown in Table 2.

\section{Table 2: PSNR to MOS Conversion}

\begin{tabular}{|c|c|}
\hline PSNR & MOS \\
\hline$>37$ & 5 (Excellent) \\
\hline $31-37$ & 4 (Good) \\
\hline $25-31$ & 3 (Fair) \\
\hline $20-25$ & 2 (Poor) \\
\hline$<20$ & 1 (Bad) \\
\hline
\end{tabular}

\subsection{Results and Discussion}

The simulation is carried out with the total number of 50 nodes. The simulations are done by acquiring PSNR value for every frames and by acquiring the average PSNR value with varied packet loss rate for three methods used for video transmission such as simple Multiple Description Coding, Routing aware MDC using SMR as a feedback for selecting the reference frames, Routing aware MDC using AOMDV for selecting the reference frames. In this work, only one video sequence has been taken. In the above figure 4 , it is shown that the PSNR value for each frame in one realization of the video with the packet loss rate of $4.5 \%$ and the comparison is made for MDC, Routing Aware MDC using SMR as routing protocol, Routing aware MDC using AOMDV as a routing protocol. The MDC method analysis in the figure 4 says that there is a drop in PSNR value when frame gets corrupted due to the packet loss in the network caused by link failure. This error propagates in the descriptions on the broken route and thus the PSNR oscillates whenever the packet loss happens in the network as the encoder uses corrupted frame as a reference frame for encoding. The RA-MDC with SMR overcomes this oscillation by making use of routing protocol (SMR) by selecting the reference frames based on the routing message and thus the error propagation gets stopped. The RAMDC with AOMDV also stops error propagation by the usage of AOMDV for routing aware purpose, but the PSNR value is found to be slightly higher than RA-MDC with SMR. From the figure 4 , the average PSNR value of RA-MDC with AOMDV is found to be $34.84 \mathrm{~dB}$, MDC is found to be 32.50 $\mathrm{dB}$ which is $2.35 \mathrm{~dB}$ less than the average PSNR value of the proposed method, RA-MDC with SMR is found to be 34.34 $\mathrm{dB}$ which is $0.50 \mathrm{~dB}$ less than average PSNR value of the proposed method. The MOS (Mean Opinion Score) of the three methods is found to be 4 , which represents the good quality of the video transmission over adhoc network. The average PSNR value of MDC up to first 50 frames is $33.793 \mathrm{~dB}$ which is found to be low as there is oscillations involved since the error propagates in the subsequent descriptions and up to 100 frames, its average PSNR value is found to be $34.19 \mathrm{~dB}$, up to 150 frames it is found to be $33.25 \mathrm{~dB}$ and up to 200 frames it is found to be $32.50 \mathrm{~dB}$ respectively. Similarly for RA-MDC using SMR, the average PSNR value up to 50 frames is $35.90 \mathrm{~dB}$, up to 100 frames it is $35.39 \mathrm{~dB}$, up to 150 frames it is $34.71 \mathrm{~dB}$ and up to 200 frames it is $34.34 \mathrm{~dB}$. The proposed method yields the average PSNR value of $36.13 \mathrm{~dB}$ up to 50 frames, $35.72 \mathrm{~dB}$ up to 100 frames, $35.32 \mathrm{~dB}$ up to 150 frames and $34.84 \mathrm{~dB}$ up to 200 frames respectively.

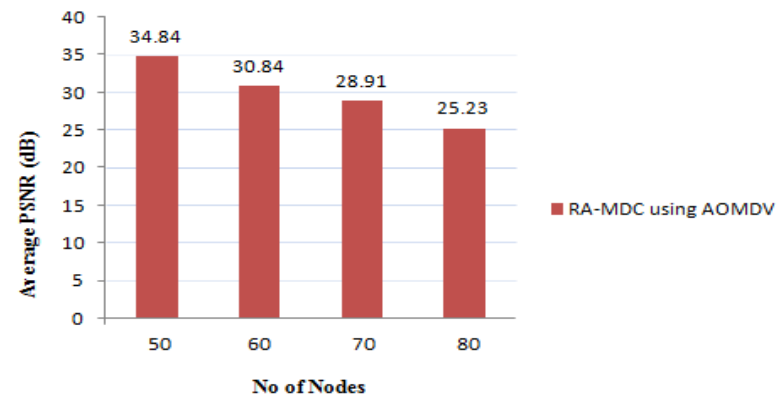

Fig 5: Analyzing Average PSNR value by varying number of nodes.

The above figure 5 shows the average PSNR value of the proposed method by varying the number of nodes with the packet loss rate of $4.5 \%$. It says that, as the number of nodes in the network increases, the network congestion increases which leads to increase in routing overhead and thus the average PSNR gets decreases. When the number of nodes involved in the ad-hoc network is 50 , it has obtained the PSNR value of $34.84 \mathrm{~dB}$ which is $4 \mathrm{~dB}$ greater than the average PSNR value of network with 60 nodes and $5.93 \mathrm{~dB}$ greater than the average PSNR value of network with 70 nodes and $9.61 \mathrm{~dB}$ greater than the average PSNR value of network with 80 nodes.

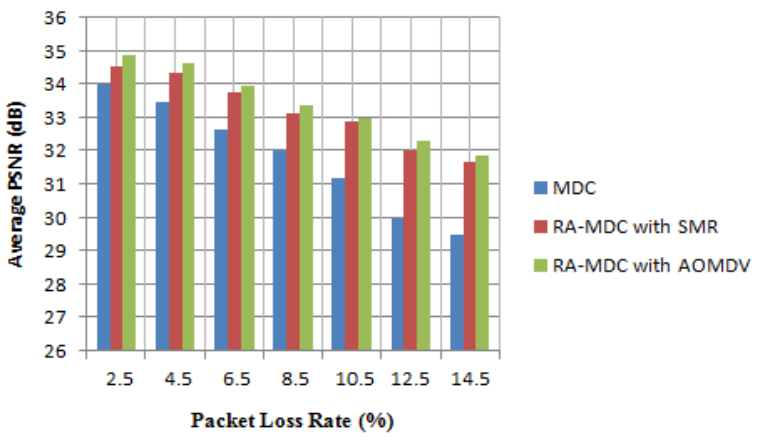

Fig 6: Comparing average PSNR of MDC, RA-MDC using SMR and RA-MD using AOMDV based on different packet loss rate

Figure 6 shows the analysis of three methods by varying the packet loss rate and by obtaining the average PSNR value. It shows that RA-MDC with AOMDV method provides slightly higher average PSNR value than RA-MDC with AOMDV for varying packet loss rate. There is a larger deviation in average PSNR value between MDC and the other two methods since in MDC, as the packet loss increases, the error propagation in the network is found to be very serious. Because unlike other 
two methods, MDC does not uses selected reference frames based on estimated packet loss happened in the network. MDC uses the corrupted frame as a reference frame and so that error propagates in the network leading to degradation in the PSNR value. Among the analysis of three methods, RAMDC with AOMDV is found to be more advantageous.

\section{CONCLUSION AND FUTURE WORK}

The proposed work is examined using NS2 simulator with EvalVid. The network is simulated for three methods such as MDC, RA-MDC using SMR and RA-MDC using AOMDV. The simulation results shows that the proposed method yields slightly higher PSNR value compare to the method that uses SMR as a routing protocol for reference picture selection yielding higher PSNR value compare to simple MDC coding method involved in the network. The MOS of the three methods is found to be 4, which represents the good quality of the video transmission over adhoc network. Then the comparative study is made on three methods by analyzing the average PSNR value for different packet loss rate. It is found that, there is a larger deviation in average PSNR value between MDC and the other two methods since in MDC, as the packet loss increases, the error propagation in the network is found to be very serious. It is also examined that, as the number of nodes increases the average PSNR value of the network gets decreases due to the congestion involved in the network. In the future work, the accuracy of the packet loss estimation can be found by varying the network conditions and also PSNR value can be found for different video files and comparison can be made by using different video standards. Also, the energy consumption at the transmitter and the receiver can also be compared along with the network lifetime.

\section{REFERENCES}

[1] Goyal, V. K., "Multiple description coding: Compression meets the network," IEEE Signal Processing Mag., vol. 18, pp. 74-93, Sep 2001

[2] Kompella, S., Mao, S., Hou, Y.T., and Sherali, H.D., "Path selection and rate allocation for video streaming in multihop wireless networks," in Proc. Military Communication Conf., pp. 1-7, Oct. 2006

[3] Begen, A.C., Altunbasak, Y., and Ergun, O., "Multi-path selection for multiple description encoded video streaming," in Proc. IEEE Int. Conf. Communications, vol. 3, pp. 1583-1589, May 2003.

[4] Yiting Liao., and Gibson, J. D., "Routing-Aware Multiple Description Video Coding Over Mobile AdHoc Networks," Proc. IEEE transactions on Multimedia, vol. 13, no. 1, February 2011.

[5] Lin, S., Mao, S., Wang, Y., and Panwar, S., "A reference picture selection scheme for video transmission over ad-hoc networks using multiple paths," in Proc. IEEE Int. Conf. Multimedia Expo., pp. 96-99, Aug 2001.

[6] Mao, S., Lin, S., Panwar, S. S., Wang, Y., and Celebi, E., "Video transport over ad-hoc networks: Multistream coding with multipath transport," IEEE J. Select. Areas Commun., vol. 21, no. 10, pp. 1721-1737, 2003.

[7] Khansari, M., Jalali, A., Dubois, E., and Mermelstein, P., "Low bit-rate video transmission over fading channels for wireless microcellular systems," IEEE Trans. Circuit Syst. Video Technol., vol. 6, pp. 1-11, Feb 1996.
[8] Liang, Y.J., Setton, E., and Girod, B., "Channel-adaptive video streaming using packet path diversity and ratedistortion optimized reference picture selection," in Proc. IEEE Workshop Multimedia Signal Processing, pp. 420 423, Dec 2002.

[9] Mahesh, K.M., and Samir, R.D., "Ad hoc on-demand multipath distance vector routing" Proceedings of Wiley InterScience Wireless Communication Mobile Computing, June 2006.

[10] Lee, S. J. and Gerla, M., "Split multipath routing with maximally disjoint paths in ad hoc networks," in Proc. IEEE Int. Conf. Communications, vol. 10, pp. 32013205, 2001

[11] Apostolopoulos, J.G., "Reliable video communication over lossy packet networks using multiple state encoding and path diversity," SPIE Proc. Visual Commun. Image Process., vol. 4310, no. 1, pp.392-409, 2001.

[12] Murthy. S., Hegde, P., Parameswaran, V., "Improved path selection algorithms for multipath video streaming in wireless ad-hoc networks," in Proc. Military Communications Conf., pp. 1-7, 2007.

[13] Wang, Y., Reibman, A.R., and Lin, S., "Multiple description coding for video delivery," Proc. IEEE, vol. 93, no. 1, pp. 57-70, Jan 2005.

[14] Heng, B.A., Apostolopoulos, J. G., and Lim, J. S., "Endto-end rate distortion optimized MD mode selection for multiple description video coding," EURASIP J. Appl. Signal Process., 2006.

[15] Liao, Y., and Gibson, J.D., "Refined error concealment for multiple state video coding over ad hoc networks," in Proc. $42^{\text {nd }}$ Asilomar Conf. Signals,Systems and computers, pp. 2243-2247, Oct 2008 .

[16] Mao, S., Hou, Y.T., Cheng, X., Sherali, H.D., Midkiff, S.F., and Zhang, Y.Q., "On routing for multiple description video over wireless ad hoc networks," IEEE Trans. Multimedia, vol. 8, no. 5, pp. 1063-1074, 2006.

[17] Gogate, N., Chung, D.M., Panwar, S.S., Wang, Y., "Supporting image and video applications in a multihop radio environment using path diversity and multiple description coding," IEEE Trans. Circuits Syst. Video Technol., vol. 12, no. 9, pp. 777-792, Sep 2002.

\section{AUTHOR'S PROFILE}

Shalini .E has received her B.E degree in Electronics and Communication Engineering from Anna University, Coimbatore, 2011. She is currently pursuing her Master of Engineering in Communication Systems in Bannari Amman Institute of Technology under Anna University, Chennai. Her areas of interest in research are Wireless Communication \& Wireless Networks. She has published 1 article in journal and 4 papers in national and international conferences.

T.V.P. Sundararajan received the BE Degree in Electronics and Communication from Kongu Engg. College, Perundurai in 1993 and the ME Degree in Applied Electronics from the Government college of technology, Coimbatore in 1999. He is Professor, working in Bannari Amman Institute of Technology, Sathyamangalam. He has published 12 articles in National and International journals and more than 25 papers in International and National conferences. He has completed his Ph.D research in Anna University, Chennai. His current research focuses are on mobile ad hoc networks and wireless security. He is member of the IEEE, ISTE and the IEEE computer society. 